\title{
O marco histórico da criação da enfermagem na universidade castelo branco
}

RESUMO | Objetivo: Descrever o processo de criação do Curso de Enfermagem na Universidade Castelo Branco. Método: A pesquisa tratou-se de um estudo histórico de 2003 a 2018 com uma abordagem qualitativa descritiva, utilizando como fontes primárias, entrevista e documentos pertencentes ao acervo do Curso de Enfermagem da Universidade Castelo Branco, localizada na Zona Oeste do Rio de Janeiro. Aplicou-se a análise de dados feita das atas de reuniões, vídeos, antecedentes históricos e eventos que constituíram os marcos históricos. Resultados: A história do Curso de Enfermagem na UCB corroborou com a formação e profissionalização do Ensino de Enfermagem na Zona Oeste RJ, contribuindo para elevar o campo da saúde, sendo assim, determinante na profissão que à época tinha certa carência de profissionais. Os cursos através das concepções pedagógicas acompanham a evolução da aquisição do saber, permitem fazer o cuidar na sua essência e desenvolvem atividades sociais que facilitam a interação entre aluno e comunidade. Conclusão: O Curso de Enfermagem da UCB apresenta um diferencial humanizado, voltado para ações sociais e educativas para a comunidade.

Palavras-chaves: História da Enfermagem; Ensino; Educação.

ABSTRACT | Objective: Describe the process of creating the Nursing Course at Castelo Branco University. Method: The research was a historical study from 2003 to 2018 with a qualitative descriptive approach, using as primary sources, interview and documents belonging to the Nursing Course collection of the Castelo Branco University, located in the West Zone of Rio de Janeiro. Data analysis of the minutes of meetings, videos, historical background and events that constituted the historical landmarks was applied. Results: The history of the Nursing Course at UCB corroborated the formation and professionalization of Nursing Education in West Zone RJ, contributing to raise the health field, thus determining the profession that at that time had a lack of professionals. The courses through the pedagogical conceptions accompany the evolution of the acquisition of knowledge, allow to take care in its essence and develop social activities that facilitate interaction between student and community. Conclusion: The UCB Nursing Course presents a humanized differential, focused on social and educational actions for the community.

Keywords: History of Nursing; Teaching; Education.

RESUMEN I Objetivo: Describir el proceso de creación del Curso de Enfermería en la Universidad Castelo Branco. Método: La investigación fue un estudio histórico de 2003 a 2018 con un enfoque descriptivo cualitativo, utilizando como fuentes primarias, entrevistas y documentos pertenecientes a la colección del Curso de Enfermería de la Universidad Castelo Branco, ubicada en la Zona Oeste de Río de Janeiro. Se aplicó el análisis de datos de las actas de reuniones, videos, antecedentes históricos y eventos que constituyeron los hitos históricos. Resultados: La historia del Curso de Enfermería en la UCB corroboró la formación y profesionalización de la Educación en Enfermería en la Zona Oeste de RJ, contribuyendo a elevar el campo de la salud, determinando así la profesión que en ese momento carecía de profesionales. Los cursos a través de las concepciones pedagógicas acompañan la evolución de la adquisición de conocimientos, permiten cuidar en su esencia y desarrollar actividades sociales que faciliten la interacción entre el alumno y la comunidad. Conclusión: El Curso de Enfermería de la UCB presenta un diferencial humanizado, enfocado en acciones sociales y educativas para la comunidad.

Descriptores:Recién Historia de la Enfermería; Enseñanza; Educación.

\section{Bruna Miguel}

Enfermeira da Universidade Castelo Branco/RJ.

Luiz Otavio Pereira dos Santos
Enfermeiro da Universidade Castelo
Branco/RJ.

\section{Maria Regina Bernardo da Silva}

Orientadora do TCC Graduação em Enfermagem Mestre em saúde da Familia UNESA, Docente da Universidade Castelo Branco RJ.

\section{Bruno Ferreira do Serrado Barbosa}

Doutor em Enfermagem UERJ.

\section{Halene Cristina Dias Armada e Silva}

Doutoranda UERJ, Diretora CMS Belizario Penna/RJ.

\section{Daniel Ribeiro soares de Souza}

Mestre em Saúde e Tecnologia UNI RIO Docente da Universidade Castelo Branco/RJ.

Recebido em: 26/05/2019

Aprovado em: 26/05/2019
INTRODUÇÃO

$\mathrm{P}$ ensar em Enfermagem, atualmente, demanda uma releitura das ideias de Florence $\mathrm{Ni}$ ghtingale, precursora da Enfermagem Moderna, as quais vêm sendo cada vez mais exploradas por diversos autores da área, que procuram entender e explicar os fundamentos de algumas questões que vivenciamos em nossa prática, principalmente, no cenário hospitalar, que permanece com um maior quantitativo de profissionais de Enfermagem ${ }^{(1)}$.

Florence Nightingale contrariou o destino de uma mulher da alta sociedade 
da época, à qual a educação e a profissão não eram permitidas, criando uma representação social e profissionalização da Enfermagem. Obteve maior visibilidade na Guerra da Criméia, em 1854, onde implementou melhorias nas enfermarias em que atuou, contribuindo para o resgate das condições sanitárias em relação ao ambiente e à higiene ${ }^{(2)}$.

A Enfermagem é uma profissão que, ao longo do tempo, vem construindo sua história, libertando-se de antigos paradigmas e introduzindo outros mais coerentes com a sua compreensão como corpo político-social e como formadora de opiniões. É também conhecida como ciência, arte e ideal de cuidar das pessoas que, segundo Florence, marcavam a diferença entre a Medicina e a Enfermagem, denominando-a como Arte de Enfermagem, com o significado de a mais bela das artes ${ }^{(2)}$.

E a história da Universidade Castelo Branco (UCB) iniciou com a fundadora do Colégio Paulo Gissoni e a Sr. ${ }^{2}$ Vera Costa Gissoni, que se formou em 1955 como Técnica de Contabilidade em Belo Horizonte e aos vinte anos teve sua primeira experiência em sala de aula, quando foi estagiar em um grupo escolar Helena Pena em Belo Horizonte. Naquele momento se tornou uma nova Vera, a Educadora formou-se em Pedagogia pela Universidade Gama Filho em 1969. De professora chegou à Reitora da Universidade, supervisionava de perto e entrevistava cada professor contratado ${ }^{(3)}$.

A UCB foi a primeira faculdade da Zona Oeste do Rio de Janeiro, tendo como contribuição para sua expansão geográfica, algumas características da sociedade contemporânea que transformaram o conceito de educação. Diante disto, criou-se a Faculdade de Educação, Ciências e Letras Marechal Castelo Branco e a Faculdade de Educação Física da Guanabara $^{(2)}$.

A Graduação em Enfermagem se deu após um aumento expressivo da procura pelo Curso Técnico em Enfermagem que existia desde 1997, e a Chanceler, em 2002, Prof. ${ }^{a}$ Vera, observou que a realida- de local associada ao reconhecimento da necessidade do mercado de trabalho para profissionais de Enfermagem, solicitou a criação de um Curso de Graduação em Enfermagem na UCB, no bairro de Realengo, segundo professora Maria Regina B da Silva ${ }^{(5)}$.

Mediante a realidade local de necessidade de cursos da Área da Saúde, o Curso de Enfermagem, então autorizado pela Resolução CEPE n. 017 de 30 de setembro de 2002, iniciou em 2003, no primeiro semestre no bairro de Realengo, Rio de Janeiro(4). Na época, o Reitor Prof. Paulo Alcântara Gomes e o Vice-reitor Acadêmico Prof. Eugenio da Silva Corrêa. Entretanto, as atividades acadêmicas do curso só se iniciaram em março de 2003 com 60 vagas semestrais, através da Resolução $n^{\circ}$ 01/2002 da Câmara Superior de Ensino (CNE/CES), de 11 de março de 2002, pelo Presidente da Câmara de Educação Superior, Arthur Roquete de Macedo. E a UCB fortalecia a sua missão norteadora na formação dos profissionais, sempre em consonância com as diretrizes curriculares nacionais ${ }^{(4)}$.

A Lei de Diretrizes e Bases da Educação Nacional ressalta que a Educação Superior tem por finalidade estimular o pensamento reflexivo dos estudantes através do desenvolvimento de pesquisas e atividades culturais, formar indivíduos capazes de atuar nas diversas áreas profissionais contribuindo no desenvolvimento social, cultural e científico, bem como suscitar na vontade de constante aperfeiçoamento $^{(4)}$.

A Enfermagem na Universidade tem como missão formar enfermeiros para atuar com competência nos diferentes âmbitos em que exerce a prática profissional, tendo como princípios uma perspectiva de educação continuada, de construção e socialização de conhecimentos $^{(6)}$. E desta forma, o Curso de Enfermagem vem com propósitos de permitir aos alunos fazerem parte, permanentemente, da construção do conhecimento, estabelecendo vínculos com a realidade e, através do olhar para o ser humano, poder desenvolver perspectivas e abordagens sobre a qualidade de vida, estabelecendo desta forma, diferenciais, com as equipes da $\cup C B^{(7)}$.

Ademais, a criação do Curso de Graduação de Enfermagem, além de grupos de pesquisa, agregou maior solidez à dimensão científica da profissão, com avanços significativos na produção de conhecimento e desenvolvimento tecnológico para a educação e para o cuidado de Enfermagem, contribuíram, desta forma, para o fortalecimento e a compreensão da profissão como força de trabalho e produtora de conhecimentos ${ }^{(8)}$.

Avaliar atualmente esta missão é verificar que a mesma permitiu ser conquistada e que possibilitou desenvolver esta pesquisa, nos inquietando saber o desenvolvimento do Curso de Enfermagem na Universidade diante de uma história de sonhos que nasceram despretensiosos e, que hoje, podem ser retratados com inúmeras fases evolutivas descritas e orientadas ao longo da pesquisa. A oportunidade de desenvolver esta pesquisa vem de encontro com um momento atual de conquista tanto da UCB como também do Curso de Enfermagem: a comemoração dos 15 anos da concepção do curso em foco.

E com este pensamento, desenvolveu-se a problemática: Como ocorreu a criação do Curso de Graduação em Enfermagem na Universidade Castelo Branco da Zona Oeste do Rio de Janeiro? O objetivo desta pesquisa é o de descrever o processo de criação do Curso de Enfermagem na Universidade Castelo Branco.

\section{METODOLOGIA}

Trata-se de um estudo histórico com uma abordagem qualitativa, tipo descritiva e documental. Considera-se como recorte temporal o início formal do ensino de Enfermagem na UCB, de 2003 a 2018. A pesquisa descritiva pode aparecer sob diversos tipos: documental, levantamentos, fotos etc. Utilizaram-se como fontes primárias, documentos per- 
tencentes ao acervo do Curso de Enfermagem da Universidade Castelo Branco localizada na Zona Oeste do Rio de Janeiro, como: filmes, fotos, documentos de reuniões e da coordenação, reitoria, secretaria, divisão institucional, informática, além de slides e entrevistas. O setor de avaliação institucional nos forneceu documentos relacionados ao número de discentes, formatura, avaliação do curso, número de docentes e documentos relativos aos coordenadores.

Os dados obtidos através da pesquisa documental foram analisados e interpretados, relacionando-os com os referenciais teóricos que tratam sobre a historiografia da Enfermagem na Universidade. Aplicou-se a análise de dados feita das atas de reuniões, vídeos, antecedentes históricos e eventos que constituíram os marcos históricos e análise temática ${ }^{(9)}$ dos documentos pesquisados, os quais foram agrupados nas seguintes categorias: A criação da Universidade Castelo Branco na Zona Oeste do Rio de Janeiro; A Enfermagem na Universidade Castelo Branco e A Universidade Castelo Branco em sua atualidade.

Por tratar-se de uma pesquisa documental e entrevista, o estudo foi submetido ao Comitê de Ética da UCB/RJ, sob n. ${ }^{\circ}$ 3.087.816 e os pesquisadores seguiram todos os preceitos éticos, segundo o que determina a Resolução n. ${ }^{\circ}$ 466/12 do Conselho Nacional de Saúde (CNS).

\section{RESULTADOS E DISCUSSÃO}

O Instituto iniciou em 1963 com crianças de bairros próximos e, no ano seguinte, se transformou no Ginásio Dr. Paulo Gissoni; em seguida, com mais expansões em um novo local na Rua Tecobé também no mesmo bairro ${ }^{(7)}$.

No ano de 1965, foram acrescentados ao Ginásio, os Cursos Científicos e Técnico em Contabilidade, fazendo a escola denominar-se em Colégio Dr. Paulo Gissoni. Em 11 de março de 1971, o Colégio passou a funcionar na Avenida Santa Cruz n. ${ }^{\circ} 1.655$, tendo seu local de origem de- sativado. Ainda no mesmo ano, criou-se o Centro de Estudos Universitários Paulo Gissoni, uma etapa muito importante para o surgimento dos primeiros cursos superiores. Em 1973, a Entidade Mantenedora recebeu um novo nome sendo denominada de Centro Educacional de Realengo, onde os primeiros cursos superiores foram autorizados a funcionar ${ }^{(7)}$.

Diante disto, criou-se a Faculdade de Educação, Ciências e Letras Marechal Castelo Branco e a Faculdade de Educação Física da Guanabara. E desta forma, a escola transformou-se no Colégio de Aplicação Dr. Paulo Gissoni ${ }^{(6)}$. Em 1990, iniciou-se o processo formal de transformação das FICAB (Faculdades Integradas Castelo BranCo), com o acolhimento da carta-consulta pelo CFE (Conselho Federal de Educação) para a criação da Universidade Castelo Branco, em 18 de fevereiro ${ }^{(8)}$.

Em sua caminhada, a UCB, constituiu um polo de incremento de cultura, esporte e capacitação profissional e até o final da década de 1980, outros cursos juntam-se aos já tradicionais, como: Letras e Educação Física, Matemática, Pedagogia, Fisioterapia, Serviço Social, Administração e Informática ${ }^{(7)}$. A instalação oficial da Universidade ocorreu no dia 4 de janeiro de 1995, após a publicação da Portaria Ministerial n. 1834 no Diário Oficial da União (DOU), no dia 29 de dezembro de 1994. Novos blocos são erguidos e o centro esportivo consolida o pioneirismo e a qualidade de ensino ${ }^{(8)}$.

A UCB tem um papel importante, além da formação acadêmica, independentemente das áreas de atuação, e reconhece que deve oferecer ao discente capacidade de identificar problemas relevantes e atuar junto à sociedade em compromisso com o exercício profissional da direção da Resolução do processo saúde e doença e cidadania ${ }^{(11)}$.

Portanto, pretende-se proporcionar a execução das ações dentro do curso de maneira sempre harmoniosa, atendendo aos objetivos que apontam para a qualidade do ensino, de maneira que atenda à evolução da sociedade e ao conceito
Universidade de forma globalizada.

A UCB firmou em sua missão a necessária formação elevada de seu corpo docente, administrativo e a qualificação de seu corpo discente; firmando-se como uma instituição de ensino, pesquisa e extensão, a qual estimula o conhecimento e a busca de soluções de problemas do mundo contemporâneo ${ }^{(7)}$. Constituiu-se como uma referência inevitável da Zona Oeste, no que diz respeito às suas instalações e Capacidade Técnica existente.

O Curso de Enfermagem na Universidade Castelo Branco

E a Zona Oeste possuía uma carência muito grande de cursos da Área de Saúde, em especial de Enfermagem; em 2002, iniciou a solicitação para um novo curso de graduação e o Curso de Enfermagem. Em março do ano de 2003, começou a primeira turma com 23 alunos e possuía carga horária de 2.620 horas com oito semestres de duração no período matutino ${ }^{(7)}$. Inicialmente, a procura pelo curso se deu por profissionais técnicos de enfermagem, funcionários da Saúde que trabalhavam nos hospitais próximos da Universidade e moradores dos arredores da Zona Oeste.

A primeira coordenadora do curso foi a Mestre Lucia Helena de Oliveira, o Reitor da Universidade era o Prof. Paulo Alcântara Gomes e o Vice-reitor Acadêmico Prof. Eugenio da Silva Correa. A Prof. ${ }^{a}$ Lucia Helena de Oliveira foi uma pessoa importante, uma vez que participou da comissão de elaboração do curso e do corpo docente inicial, que na época de criação já lecionava e demonstrava um leque de conhecimentos e experiência no campo da educação.

O corpo docente do Curso de Enfermagem no ano de 2003, formado por onze docentes, sendo um doutor, seis mestres e quatro especialistas, além da professora Lúcia Helena de Oliveira, alguns professores que muito contribuíram no início do curso: Ana Cristina Casagrande Vianna, Jorge Teixeira de Azevedo, Maria Aparecida Thiengo, Marília 
Teixeira Moraes dos Santos, Sandra Rosana Correa e Vaneir Inocêncio Bezerra. Em 2003, existiam no Campus Realengo os laboratórios de: Ciências Biológicas, Informática, Multimeios, Laboratório da Escola Técnica, Citologia, Histologia e também Anatomia que também atendia ao CAP na formação dos profissionais técnicos de Enfermagem ${ }^{(5)}$.

Fatos marcantes no curso ocorreram neste período como a Aula Magna em abril de 2003 com a participação da Dra Maria Antonieta Rubio Tyrrel, Professora da UFRJ e foi diretora do Coren-RJ, abordando o assunto sobre Atividades do Enfermeiro, tema de extrema importância para os discentes, a qual foi a primeira divulgação externa do curso e alguns discentes de cursos de saúde foram convidados a participar. A Primeira Semana de Enfermagem foi bem expressiva com a Dra. Almerinda Moreira, que foi Diretora da UNIRIO e Professora de História de Enfermagem, cujo tema abordado foi "A origem da profissão de Enfermagem"(5).

O curso no término do ano de 2003 já começando a ser conhecido pelos profissionais que trabalhavam nos hospitais próximos a instituição e demais categorias profissionais. No ano de 2004, a Enfermeira Dr ${ }^{\mathrm{a}}$. Ângela Maria e Silva assumiu a coordenação e, na época, com a coordenadora adjunta, a Mestre Maria Regina Bernardo da Silva inauguraram um novo Laboratório de Enfermagem para o Curso de Graduação, que também atendia ao CAP na formação dos profissionais técnicos de Enfermagem. Esse laboratório tinha duas amplas salas e uma boa estrutura para desenvolvimento das técnicas dos Cursos de Enfermagem ${ }^{(5)}$.

Nesta época, outro fato marcante no curso ocorreu devido a Enfermagem ter sido reconhecida pela mídia, na primeira ação social feita com os acadêmicos na UCB Campus Penha no Curso de Veterinária em outubro de 2004, no qual o coordenador na época era Dr. Marcelo Hauaji de Sá Pacheco ${ }^{(5)}$.

Os discentes participaram com atividades preventivas, aferiram pressão arte- rial, fizeram ações educativas de IST e Hipertensão Arterial para os acadêmicos da universidade, funcionários e comunidade.

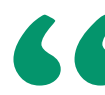

\section{Inicialmente,} o curso tinha 60 vagas para discentes ingressantes $\mathrm{e}$ permaneceu assim até o ano de 2005 (período 2005.1), quando houve um aumento para 100 vagas. fato para esse aumento foi devido a Universidade aderir aos Programas e Políticas Públicas na Área da Educação, com isso, ampliando a procura pela rede privada $^{(7)}$.

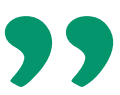

Inicialmente, o curso tinha 60 vagas para discentes ingressantes e permaneceu assim até o ano de 2005 (período 2005.1), quando houve um aumento para
100 vagas. $O$ fato para esse aumento foi devido a Universidade aderir aos Programas e Políticas Públicas na Área da Educação, com isso, ampliando a procura pela rede privada $^{(7)}$.

Os programas de crédito educativo foram fundamentais para o aumento do número de vagas na rede privada de ensino superior, concedendo aos estudantes o financiamento dos seus estudos. $\mathrm{O}$ Fundo de Financiamento ao Estudante do Ensino Superior (FIES), regulamentado pela Portaria MEC n. ${ }^{\circ}$ 860/2003, substituiu o Programa de Crédito Educativo (CREDUC) instituído em 1976. Mas foi implantando no governo Luís Inácio Lula da Silva o Programa Universidade para Todos (PROUNI), que fornecia a bolsa de estudos aos estudantes de baixa renda ${ }^{(11)}$.

Em seguida, em 2005, a Professora Enfermeira Cristiane Costa Miranda assumiu a Coordenação adjunta com a Dra Ângela Maria e Silva e houve mudanças no Projeto Pedagógico com aumento de carga horária do curso e vários professores participaram, como: Antônia Regina Messias de Sena, Brígida Neide Rocha Lira, Cristiane Duarte, Maria Regina Bernardo da Silva, Sandra Rosana Correa, Sandro Ribeiro Fernandes, Sonia Sueli do Espirito Santo e Wagner Luiz Natario ${ }^{(5)}$.

Neste ano, o Curso de Graduação em Enfermagem funcionando nos turnos manhã e noite, com 289 alunos, foi reconhecido pela comunidade pela sua excelência e qualidade na Zona Oeste, dados fornecidos pela divisão de ensino em 2005.

Em 2005, ocorreu também uma visita marcante em uma emissora de televisão com a professora Sonia Sueli do Espírito Santo na disciplina Saúde do Trabalhador. Este fato foi transmitido pela Rede de televisão, demonstrando ações educativas realizadas com os funcionários da empresa, sendo muito gratificante para os acadêmicos de enfermagem que ficaram muito encantados em participar da ação e interagir com os funcionários através de educação em saúde ${ }^{(5)}$.

Foi idealizado um novo projeto po- 
lítico pedagógico com 4000 horas, em que as disciplinas foram ministradas nos períodos matutino, vespertino e noturno com um regime acadêmico de créditos semestrais, seu tempo de integralização curricular podendo variar de oito períodos no mínimo e doze no máximo; a relação professor-aluno em aulas teóricas foi de cinquenta alunos, esta proporção em aulas práticas foi de vinte e cinco alunos, tornando-se vital uma adequação no campo de estágio supervisionado em que, um preceptor para dez alunos e, em áreas críticas, um preceptor para seis alunos ${ }^{(12)}$.

O Curso de Graduação em Enfermagem apresentando aumento expressivo de discentes em março de 2006, a Dra. Ana Paula de Carvalho Orichio, a terceira coordenadora do curso, idealizou as propostas do curso, sendo bastante valorizado, elevando sua carga horária para 4100 horas, e que está até o presente PPP, juntamente com alguns professores enfermeiros que foram decisivos na manutenção da qualidade do ensino, como: Alcinéa Cristina Ferreira de Oliveira, Cláudia Luiza F. Orofino, Bruno da Silva Lourenço, Claudia Angélica M. F. Merces, Cláudia Maria Messias, Halene Cristina Armada Dias e Silva, Márcia de Deus Gomes, Raquel de Mendonça Nepomuceno, Rafael Celestino as Silva, Solange Cassiano Borges, Theia Maria Forney e Wanderley Castellos.
Neste ano, o Secretário de Educação Superior, usando da competência que lhe foi delegada pelo Decreto n. ${ }^{\circ} 5.773$, de 9 de maio de 2006, resolveu através do Artigo $1^{\circ}$ : "reconhecer os cursos superiores de graduação, ministrados pelas instituições de ensino superior como o curso de Enfermagem, nível Bacharelado, com a Mantenedora sendo o Centro Educacional de Realengo e localidade no estado do Rio de Janeiro"

A primeira turma formou-se em dezembro de 2006, sendo a formatura oficializada em fevereiro de 2007 com um quantitativo de dez discentes. São eles: Ana Carolina Garcia de Souza, Fernando dos Santos Arruda, Ellen Fernandes Brum, Marciele Gottgtroy Gonçalves, Maria Cristina da Silva Pereira, Paulo Henrique Ferreira Ribeiro, Queila Ferreira Vidal, Rozana de Araújo Valverde, Tatiane Cristina Tavares da Silva e Vanessa Marques de Góes ${ }^{(5)}$.

A Clínica Escola Castelo Branco teve um papel fundamental no aprimoramento dos profissionais de Enfermagem, em formação na universidade onde atuam em atividades sociais que facilitam o atendimento da comunidade, com ações de Educação e Saúde, imunização e atividades individuais e coletivas.

A Clínica Escola, desde 2006, é um grande potencializador, onde os discentes e docentes participam de ações sociais, campanhas de vacina, imunizando os colaboradores, os discentes e a comunidade usuária da Clínica Escola e, também avaliação de risco de pacientes diabéticos, grupo de hipertensos, avaliação e orientações aos funcionários sobre Equipamento de Proteção Individual (EPI). E o Curso de Enfermagem apresenta um diferencial humanizado, voltado para ações sociais e educativas para a comunidade ${ }^{(5)}$.

Em 2007, aconteceu a primeira avaliação do curso realizada pelo ENADE (Exame Nacional de Desempenho de Estudantes) com conceito 2.0. Este conceito se deu devido ao fato de que alguns discentes apresentarem dificuldades em não entender a importância desta avaliação para o curso, para a universidade e para eles mesmos tendo assim uma baixa adesão à prova, no entanto o conceito do curso (CC) foi cinco - 05 $5^{(11)}$.

Em 2010 e 2013, continuou o ENADE com nota dois, mas o conceito preliminar do curso (CPC) foi nota três e, somente em 2016, o Curso de Enfermagem conseguiu nota três no ENADE e três no Conceito Preliminar do Curso. Os conceitos referentes ao Índice Geral de Curso (IGC), Conceito Preliminar de Curso (CPC), Exame Nacional de Desempenho de Estudantes (ENADE), Indicador de Diferença entre Desempenhos Observado e Esperado (IDD) e Conceito de Curso (CC) estão apresentados na tabela abaixo:

Tabela 1. Históricos dos índices do curso de Enfermagem - Educação presencial. Rio de Janeiro, RJ, Brasil, 2018

\begin{tabular}{|c|c|c|c|c|}
\hline ANO & ENADE & $\mathrm{CPC}$ & $\mathrm{CC}$ & IDD \\
\hline 2013 & 2 & 3 & - & - \\
\hline 2010 & 2 & 3 & - & - \\
\hline 2007 & 2 & 2 & - & 3 \\
\hline 2006 & - & - & 5 & - \\
\hline
\end{tabular}

Fonte: Avaliação Institucional UCB de 2010.

O Ministério da Educação (MEC), em 2012, realizou o primeiro Relatório de Reconhecimento e Renovação do Curso, com o objetivo de verificar se o Projeto Político Pedagógico apresentado estava sendo cumprido adequadamente. Nesse relatório, foram avaliadas três dimensões, sendo elas: organização didático-pedagógica; corpo docente, corpo discente e corpo técnico-administrativo e instala- ções físicas. O Curso de Enfermagem recebeu nota três ${ }^{(11)}$.

A partir de 2012, o Curso de Graduação em Enfermagem passou a apresentar dez períodos e a carga horária de 4.100 
horas, funcionando nos turnos manhã e noite, e os estágios sendo realizados no $9^{\circ}$ e $10^{\circ}$ períodos. E o curso a cada dia com melhores qualificações profissionais, com projetos de extensão e pós-graduação ${ }^{(12)}$.

E o quarto coordenador, Mestre Bruno da Silva Lourenço, que já era coordenador adjunto, assumiu a coordenação geral trazendo inovações com aulas com maior infraestrutura, laboratórios específicos para aulas práticas e aulas de monitoria. E professores importantes fazendo parte deste novo contexto do Curso de Enfermagem da UCB, são eles: Adriana Loureiro da Cunha Santos, Bruno Ferreira do Serrado Barbosa, Daniel Ribeiro, Claudia da Silva Medeiros e Leandro Andrade da Silva ${ }^{(5)}$.

E o curso mantendo a qualidade e com grande procura devido as políticas públicas de incentivo a permanência no Ensino Superior, e melhoria no acesso à educação superior das minorias ${ }^{(11)}$.

No ano de 2014, o curso completando dez anos, segundo a avaliação institucional 412 já tinham colado grau e tinham 678 alunos matriculados ${ }^{(14)}$. E devido as necessidades e cobranças acontecendo com maior frequência, foram reformuladas com metodologias ativas, enfoque voltado para formação profissional com ensino pesquisa e extensão através do quinto e atual coordenador do curso Dr. Bruno Ferreira do Serrado Barbosa e a Coordenadora adjunta Ana Claudia Lopes de Almeida. Atualmente, o curso tem um pós-doutor, três doutores, dois doutorandos, nove mestres, três mestrandos e cinco especialistas ${ }^{(5)}$.

Neste sentido, o Curso de Enfermagem programa concepções pedagógicas que acompanham a evolução da aquisição do saber, sentir e fazer no cuidar. A estrutura curricular do curso está delineada para uma concepção pedagógica direcionada a novas formas de construção de conhecimentos para o campo profissional, com competência, habilidade e autonomia pautadas em princípios éticos e de cidadania, respaldada tanto pelas leis que regem o ensino quanto pelas que são específicas dos órgãos reguladores na área do conhecimento.

\section{CONCLUSÃO}

A pesquisa sobre o marco histórico da Enfermagem na Universidade Castelo Branco permitiu considerar que o Curso se apresentou importante para a formação e profissionalização do Ensino de Enfermagem no Rio de Janeiro em especial à Zona Oeste, contribuindo para elevar o campo da saúde. Além disso, foi determinante para o fortalecimento local de uma profissão, que na época com certa carên- cia de profissionais, com atuação ética e a prestação de cuidados com qualidade aos usuários da comunidade.

Esta Universidade ratifica a tradição de meio século de bons serviços prestados à educação, com a cooperação de uma equipe profissional docente selecionada e de qualidade comprometida, e sempre acreditando que os melhores resultados na educação provêm do investimento constante na qualidade do ensino e na crença dos sonhos do seu corpo discente. E tem um papel importante, além da formação acadêmica, independentemente das áreas de atuação, e reconhece que deve oferecer ao discente a capacidade de identificar problemas relevantes e atuar junto à sociedade em compromisso com o exercício profissional da direção da Resolução do processo saúde e doença e cidadania.

Desta forma, a Enfermagem na Universidade Castelo Branco foi se desenvolvendo com metas para que os discentes possuíssem a possibilidade de fazer parte permanentemente da construção do curso com um diferencial humanizado, voltado para ações sociais e educativas com a comunidade, estabelecendo vínculos com a realidade.

Agradecimento aos funcionários dos setores: Biblioteca, Multimeios, Informática e Avaliação Institucional da Universidade Castelo Branco, em especial ao Sr. Danilo Azevedo.

\section{Referências}

1. Gottems LBD. A história da educação e do ensino de enfermagem e as ressonâncias na prática do cuidado em enfermagem e saúde. Universidade Católica de Brasília e da Escola Superior de Ciências da Saúde do Governo do Distrito Federal. HIST. ENF. REV. ELETR (HERE). 2014.

2. Malagutti W, Miranda SMRC. Os caminhos da Enfermagem: de Florence à globalização. São Paulo: Phorte Editora; 2010.

3. Padilha MICS, Nazário NO, Moreira MC. A compreensão do ideário da enfermagem para a transformação da prática profissional. Rev. Bras Enferm. 1997; 50(3):307-322.

4. LDB. Lei de Diretrizes e Bases da Educação Nacional. $10^{\text {a }}$ edição. Câmara; 54 Legislatura. $4^{\text {a }}$ Sessão Legislativa. 2015.Capitulo IV do Ensino da Educação Superior Art.43 Inciso 1.

5. Silva MRB. Entrevista sobre implantação da UCB. 2018.

6. Universidade Castelo Branco. Projeto Pedagógico - Curso de Enfermagem. UCB, RJ, 2005.

7. Julião L. História da Universidade Castelo Branco. Revista Universidade Castelo Branco 15 anos. Coordenação ICOM; UCB/RJ. 12/2009.
8. Conselho Nacional de Educação, Câmara de Educação Superior (BR). Resolução CNE/CES n. ${ }^{\circ}$ 3, de 7 de novembro de 2001. Institui diretrizes curriculares nacionais do curso de graduação em enfermagem. Comissão institucional das faculdades integradas castelo branco (FICAB), 2005. Resolução CEPE n. 017 de 30 de setembro de 2002.

9. Minayo MCS. 0 desafio do conhecimento: pesquisa qualitativa em saúde. 12 ed. São Paulo: Hucitec; 2010.

10. Finger AP, Leire HML, Braga R. Equipe técnica pedagógica. Comissão Institucional das Faculdades Integradas Castelo Branco, 2006.

11. Universidade Castelo Branco. Plano de Desenvolvimento da Universidade Castelo Branco - PDI 2012 a 2016.

12. Ministério da Educação e Cultura (BR). Lei n. ${ }^{\circ} 10.172$ de 2001. Plano Nacional da Educação, 2008.

13. Universidade Castelo Branco. Projeto Pedagógico- Curso de Enfermagem UCB, RJ, 2012.

14. Biblioteca Manuel Bandeira. Universidade Castelo Branco. Histórias da UCB. Rio de Janeiro, 2014. 